Discussiones Mathematicae

Graph Theory 29 (2009) 87-99

\title{
DECOMPOSITIONS OF QUADRANGLE-FREE PLANAR GRAPHS
}

\author{
OleG V. BORODIN* \\ Sobolev Institute of Mathematics \\ Novosibirsk 630090, Russia \\ e-mail: brdnoleg@math.nsc.ru \\ AnNA O. IvanOva ${ }^{\dagger}$ \\ Yakutsk State University \\ Yakutsk, 677000, Russia \\ e-mail: shmgnanna@mail.ru \\ Alexandr V. KostochKA \\ Department of Mathematics \\ University of Illinois, Urbana, IL 61801, USA \\ and \\ Sobolev Institute of Mathematics \\ Novosibirsk 630090, Russia
}

e-mail: kostochk@math.uiuc.edu

AND

Naeem N. Sheikh

Department of Mathematics

University of Illinois, Urbana, IL 61801, USA

e-mail:nsheikh@math.uiuc.edu

\footnotetext{
${ }^{*}$ Research supported in part by the RFBR grants 08-01-00673 and 06-01-00694.

${ }^{\dagger}$ Research supported in part by the RFBR grants 08-01-00673 and 06-01-00694.

${ }^{\ddagger}$ Research supported in part by NSF grant DMS-0650784 and the RFBR grant 06-0100694 .
} 


\begin{abstract}
W. He et al. showed that a planar graph not containing 4-cycles can be decomposed into a forest and a graph with maximum degree at most 7. This degree restriction was improved to 6 by Borodin et al. We further lower this bound to 5 and show that it cannot be improved to 3 .
\end{abstract}

Keywords and phrases: planar graphs, graph decompositions, quadrangle-free graphs.

2000 Mathematics Subject Classification: 05C15, 05C10, 05C35.

\title{
1. INTRODUCTION
}

He, Hou, Lih, Shao, Wang and Zhu [5] proved a number of results on decomposing a planar graph with specified girth conditions into a forest and another graph whose maximum degree is not too high. In the same paper, they also used these results to derive new upper bounds on the game chromatic number of graphs.

In particular, He et al [5] showed that a planar graph with girth $g$ can be decomposed into a forest and a graph $H$ such that the maximum degree, $\Delta(H)$, of $H$ is at most 4,2 or 1 if $g$ is at least 5,7 or 11 , respectively. Kleitman [6] improved one of these results by showing that $\Delta(H) \leq 2$ if $g \geq 6$. Kleitman et al. [1] also proved that $\Delta(H) \leq 1$ when $g \geq 10$. This result was further improved by Borodin, Kostochka, Sheikh and Yu [3]: $\Delta(H) \leq 1$ if $g \geq 9$.

In addition to the results with girth conditions, He et al. [5] proved that a planar graph that does not contain 4-cycles (even though it may contain 3cycles) can be decomposed into a forest and a graph $H$ such that $\Delta(H) \leq 7$. This bound was improved to 6 by Borodin et al [4].

Our main result here is as follows.

Theorem 1. Every planar graph without 4-cycles can be decomposed into a forest and a graph with maximum degree at most 5 .

The reason for forbidding 4-cycles is seen in the complete bipartite graph $K_{2, n}$, which is planar. Any forest $F$ contained in $K_{2, n}$ has at most $n+1$ edges, and at least half of the remaining edges are incident with one of the two vertices of degree $n$ in $K_{2, n}$. Thus, in any decomposition of $K_{2, n}$ into $F$ and another graph, $H$, the maximum degree of $H$ is at least $\frac{n-1}{2}$, i.e., is 
high if $n$ is large. On the other hand, forbidding only 2-alternating 4-cycles (having two nonconsecutive vertices of degree 2) in a planar graph implies that this graph can be decomposed into a forest and an $H$ with $\Delta(H) \leq 14$ (by Theorem 5 in [2] combined with Theorem 3.1 in [5]).

As explained in [5], Theorem 1 implies the following result.

Corollary 2. The game chromatic number and the game coloring number of a planar graph without 4-cycles are at most 9 .

By an $\mathrm{FH}_{k}$-coloring of a graph we mean a partition of its edges into a forest $F$ and a graph $H$ of maximum degree at most $k$, i.e., a coloring of the edge set of the graph with two colors, $F$ and $H$, such that the set of edges colored $F$ forms a forest and the set of edges colored $H$ forms a graph of maximum degree at most $k$.

\section{Proof of Main Result}

Say that a vertex is branching if its degree is at least 3. Suppose that there exist counterexamples to Theorem 1 . In the set of these counterexamples, consider the subset that contains graphs with the fewest branching vertices. Let $G$ be a graph from this subset with the smallest total number of vertices and edges.

\subsection{Basic structural properties of $G$}

Claim 3. $G$ is connected.

Fix a planar embedding of $G$ and let $F(G)$ denote the set of faces of $G$ in this planar embedding. For $x \in V(G) \cup F(G), d(x)$ denotes the degree of $x$, where the degree of a face is the length of a closed walk around the boundary of the face.

Claim 4. $G$ has no vertex $v$ with $d(v)=1$.

Proof. Let $G^{\prime}=G-v$. By the minimality of $G$, graph $G^{\prime}$ has an $F H_{5^{-}}$ coloring, and it suffices to color the edge incident with $v$ with $F$ to obtain an $\mathrm{FH}_{5}$-coloring of $\mathrm{G}$.

Claim 5. Every edge in $G$ is incident with a vertex of degree at least 7 . 
Proof. Suppose $u$ and $v$ are two adjacent vertices of degree at most 6 . Let $G^{\prime}=G-u v$. Since the number of branching vertices in $G^{\prime}$ does not exceed that in $G$, and $G^{\prime}$ has fewer edges than $G, G^{\prime}$ has an $F H_{5}$-coloring. If both $u$ and $v$ have incident edges of color $F$, then we color $u v$ with $H$; otherwise, color it with $F$. This gives an $F H_{5}$-coloring of $G$, a contradiction.

Claim 6. $G$ has no vertices of degree 3 .

Proof. Suppose that $G$ has a vertex $v$ of degree 3 , with neighbors $v_{1}, v_{2}$, and $v_{3}$. Let $G^{\prime}$ be obtained from $G$ by removing $v$, adding three new vertices $x_{1}, x_{2}$, and $x_{3}$, and the edges of the 6-cycle $v_{1} x_{1} v_{2} x_{2} v_{3} x_{3}$. By Claim 5, $G^{\prime}$ has fewer branching vertices. Thus by the minimality of $G, G^{\prime}$ has an $\mathrm{FH}_{5^{-}}$ coloring $c^{\prime}$. We use $c^{\prime}$ to construct an $F H_{5}$-coloring $c$ for $G$ : we let $c\left(v v_{i}\right)=F$ if both new edges at $v_{i}$ in $G^{\prime}$ are colored $F$, and let $c\left(v v_{i}\right)=H$ otherwise. Then $v$ has at most three incident edges colored $H$, the number of edges colored $H$ in $G$ incident with any vertex $v_{i}$ does not exceed that in $G^{\prime}$, and no $F$-path in $G$ going through $v$ can appear.

For a vertex $v$, let $d^{+}(v)$ denote the total number of adjacent vertices of degree 2 and triangular faces incident with $v$.

Claim 7. $G$ has no vertex $v$ such that $d^{+}(v)>d(v)$.

Proof. We first note that by Claim 5, each closed walk $\left(w_{1}, \ldots, w_{t}\right)$ encounters at least $\left\lceil\frac{t}{2}\right\rceil$ vertices of degree at least 7 . Suppose that the neighbors of $v$ in the clockwise direction are $w_{1}, \ldots, w_{t}$. Let $f_{i}$ be the face containing the walk $\left(w_{i}, v, w_{i+1}\right), i=1, \ldots, t$. Some vertices and/or faces with distinct labels can coincide. If a face $f_{i}$ is a triangle, then by the first sentence of this proof, at least one of $w_{i}$ and $w_{i+1}$ is a vertex of degree at least 7 . Call such a vertex $x_{i}$. Since $G$ has no 4-cycles, all $x_{i}$ are distinct. Thus the number of neighbors of degree at least 7 is at least the number of the incident triangular faces. Hence, the total number of adjacent 2-vertices and incident triangular faces is at most the number of adjacent vertices of degree 2 plus the number of adjacent vertices of degree at least 7 , which is at most the degree of $v$.

\subsection{Discharging and its consequences}

Let the initial charge of every $x \in V(G) \cup F(G)$ be $\mu(x)=d(x)-4$. By Euler's formula $|V(G)|-|E(G)|+|F(G)|=2$, we have 


$$
\sum_{x \in V(G) \cup F(G)}(d(x)-4)=\sum_{x \in V(G) \cup F(G)} \mu(x)=-8<0 .
$$

A face $f$ of degree at least 5 is weak if it is incident with $d(f)-3$ vertices of degree 2. Non-triangular faces that are not weak will be called strong. By Claim 5, every face $f$ with $d(f) \geq 7$ is strong.

The vertices and faces of $G$ discharge their initial charges by the following rules:

Rule 1. Every non-triangular face $f=u v w \ldots$ gives each incident vertex $v$ of degree 2 :

(a) 1 , if either $f$ is strong or $f$ is a weak 6 -face adjacent to the 3 -face $u v w$;

(b) $\frac{1}{2}$, otherwise.

Rule 2. Every triangle gets $\frac{1}{2}$ from every incident vertex of degree at least 7 .

Rule 3. Every vertex $w$ of degree at least 7 gives $\frac{1}{2}$ to every adjacent 2vertex $v$, unless $v$ lies between non-triangular faces at least one of which is strong, in which case $w$ gives only $\frac{1}{4}$ to $v$.

In the rest of the paper, we show that the final charge $\mu^{*}(x)$ is nonnegative for each $x \in V(G) \cup F(G)$, which is a contradiction to (1) since the total charge is preserved.

If $f$ is a 3 -face, then $\mu^{*}(f) \geq 3-4+2 \times \frac{1}{2}=0$ by Rule 2 , due to Claim 5 . By Rule 1 combined with Claim 5, each 5 -face ends with a charge of at least 0 . For the same reasons, each 6 -face $f$ has $\mu^{*}(f) \geq 6-4-2 \times 1=0$ if $f$ is strong and $\mu^{*}(f) \geq 6-4-2 \times \frac{1}{2}-1=0$ if $f$ is weak. (Note that a weak 6 -face cannot give 1 to more than one incident 2 -vertex due to the absence of 4-cycles in $G$.) Finally, if $d(f) \geq 7$, then $\mu^{*}(f) \geq d(f)-4-\left\lfloor\frac{d(f)}{2}\right\rfloor \times 1 \geq 0$.

Now suppose $v \in V(G)$. If $d(v)=2$, then $\mu(v)=-2$. Note that $v$ cannot be incident with two triangle faces, since $G$ has no multiple edges. If one of the faces, $f_{1}$, incident with $v$ is a triangle, then the other, $f_{2}$, cannot be a weak 5 -face, and hence $\mu^{*}(v) \geq 2-4+1+2 \times \frac{1}{2}=0$ by Rules 1 and 3 . If both $f_{1}$ and $f_{2}$ are non-triangular, then $\mu^{*}(v) \geq 2-4+1+\frac{1}{2}+2 \times \frac{1}{4}=0$ provided that at least one of $f_{1}, f_{2}$ is strong; otherwise, $\mu^{*}(v) \geq 2-4+4 \times \frac{1}{2}=0$.

If $4 \leq d(v) \leq 6$, then $\mu^{*}(v)=\mu(v)=d(v)-4 \geq 0$. If $d(v) \geq 8$, then $\mu^{*}(v) \geq d(v)-4-d^{+}(v) \times \frac{1}{2} \geq d(v)-4-d(v) \times \frac{1}{2}=\frac{d(v)-8}{2} \geq 0$ by Claim 7. Vertices of degree 7 are considered in the next subsection. 


\subsection{Handling vertices of degree 7}

Note that $d^{+}(v) \leq 6$ implies $\mu^{*}(v) \geq 7-4-6 \times \frac{1}{2}=0$, so we can assume $d^{+}(v)=7$ due to Claim 7. There are four configurations to consider.

Let the neighbors of $v$ be labeled $v_{1}, \ldots, v_{7}$ in the clockwise order.

Configuration 1. Vertex $v$ is adjacent to seven 2-vertices and not incident with any triangle.

Let the neighbor of $v_{i}, i \in\{1, \ldots, 7\}$, other than $v$ be labeled $w_{i}$. Note that each $w_{i}$ is of degree at least 7 , and that they are all distinct, since $G$ has no 4-cycles.

Let $G_{i, j}$ be the graph obtained from $G$ by removing $v$ and all 2-vertices adjacent to it, adding a new vertex $x$ and the edges $w_{i} x$ and $w_{j} x$. Note that $G_{i, j}$ is still planar and has fewer branching vertices than $G$. If $G_{i, j}$ is $C_{4}$-free, then by the minimality of $G$, there exists an $F H_{5}$-coloring $c^{\prime}$ of $G_{i, j}$. We show how to extend $c^{\prime}$ to an $F H_{5}$-coloring $c$ of $G$.

We let $c\left(w_{l} v_{l}\right)=c^{\prime}\left(w_{l} x\right)$ for $l=i, j$ and $c\left(v_{i} v\right)=c\left(v_{j} v\right)=F$, while $c\left(v_{l} v\right)=H$ and $c\left(w_{l} v_{l}\right)=F$ for $l \neq i, j$. Note that if $c^{\prime}\left(w_{i} x\right)=c^{\prime}\left(w_{j} x\right)=F$, then there is no other $F$-path from $w_{i}$ to $w_{j}$ in $G_{i, j}$ and hence the $F$-path $w_{i} v_{i} v v_{j} w_{j}$ does not produce any $F$-colored cycle in coloring $c$ of $G$.

Thus, we may assume that for each $i \neq j, i, j \in\{1, \ldots, 7\}$, graph $G_{i, j}$ has a 4 -cycle, i.e., $w_{i}$ has a common neighbor with $w_{j}$.

Claim 8. Let $G^{\prime}$ be obtained from $G$ by deleting $v$ and all its neighbors of degree 2. If every pair of vertices from the set $\left\{w_{1}, \ldots, w_{7}\right\}$ in $G^{\prime}$ has a common neighbor, then there exists a vertex distinct from these seven vertices that is adjacent to all of them.

Proof. Case 1. First suppose that there is a chord of type $w_{i} w_{i+3}$ for some $i$ in $G^{\prime}$, say, $w_{1} w_{4}$. There are two possibilities: (a) neither edge $w_{1} w_{3}$ nor edge $w_{2} w_{4}$ is present, and (b) exactly one of them exists (both cannot be present by planarity). If (a) holds, then for $w_{2}$ to have a common neighbor with $w_{5}$, the edges $w_{1} w_{2}$ and $w_{1} w_{5}$ must be present, and similarly in order for $w_{3}$ to have a common neighbor with $w_{6}$ the edges $w_{3} w_{4}$ and $w_{4} w_{6}$ must be present. But by planarity, edges $w_{1} w_{5}$ and $w_{4} w_{6}$ cannot be present at the same time.

Suppose now that (b) holds; say, $w_{1} w_{3} \in E(G)$. In order for $w_{2}$ to have a common neighbor with any of the vertices in the set $\left\{w_{5}, w_{6}, w_{7}\right\}$, the edge $w_{1} w_{2}$ must exist. If both edges $w_{2} w_{3}$ and $w_{3} w_{4}$ exist, then the 4 -cycle 
$w_{1} w_{2} w_{3} w_{4} w_{1}$ arises. Thus, one of these two edges does not exist. If the edge $w_{2} w_{3}$ is not there, then the face containing $w_{2}, w_{3}$ and $v$ in the original graph $G$ is strong. Indeed, it either contains $w_{1}$, in which case it is a strong 6 -face, or is a face with at least 7 edges (if there is a $w_{2}$ to $w_{3}$ path, it must have at least 3 edges, since 2 edges would produce a 4 -cycle with the edges $w_{1} w_{2}$ and $\left.w_{1} w_{3}\right)$. Note that the presence of a strong face at $v$ ensures that $\mu^{*}(v) \geq 0$. Similarly, if the edge $w_{3} w_{4}$ is absent, then the face incident with $w_{3}, w_{4}$ and $v$ in $G$ is strong. Thus, there is no chord of type $w_{i} w_{i+3}$ if $v$ has a negative final charge.

Case 2. Now suppose there is no chord of the kind $w_{i} w_{i+3}$ but there is a chord of the kind $w_{i} w_{i+2}$, say, $w_{1} w_{3}$. Since $w_{3} w_{6} \notin E(G)$, to have a path of length 2 from $w_{6}$ to $w_{2}$, there should be edges $w_{1} w_{6}$ and $w_{1} w_{2}$. Similarly, to have a path of length 2 from $w_{2}$ to $w_{5}$, the edges $w_{2} w_{3}$ and $w_{3} w_{5}$ should be present. Now, because of planarity, there cannot be a path of length 2 between $w_{4}$ and $w_{7}$, a contradiction.

Case 3. Finally, suppose there are no chords in this set of vertices. To produce a path of length 2 from $w_{1}$ to $w_{4}$, there should be a common neighbor $w$ of $w_{1}$ and $w_{4}$. Also, there must be a common neighbor $w^{\prime}$ of $w_{2}$ and $w_{6}$. By planarity, $w^{\prime}$ must be the same vertex as $w$. Similarly, $w_{5}$ must also be adjacent to $w$, and in fact all the other vertices must be adjacent to $w$ to get all the paths of length 2 .

By Claim 8, we can assume that there is a vertex $w$ adjacent to each $w_{i}$. Since $G$ has no 4-cycles, either $w_{1} w_{2} \notin E(G)$ or $w_{2} w_{3} \notin E(G)$. Suppose that $w_{1} w_{2} \notin E(G)$. Then the face incident with $v, v_{1}$ and $v_{2}$ is strong. Hence, $\mu^{*}(v) \geq 0$.

Configuration 2. Vertex $v$ is adjacent to six 2-vertices and incident with one triangle.

Let $v_{7}$ have degree larger than 2, and let the triangle incident with $v$ contain $v_{7}$ and $v_{6}$. Let the neighbors of $v_{i}, i=1, \ldots, 6$, be labelled $w_{i}$; in particular, $v_{7}=w_{6}$. Like in Configuration 1 above, consider $G_{i, j}$ for $1 \leq i<j \leq 6$ (we do not delete $v_{7}$ ). If some $G_{i, j}$ is $C_{4}$-free, then by the minimality of $G$ as a counterexample, there exists an $F H_{5}$-coloring $c^{\prime}$ of $G_{i, j}$. We will show that one can extend $c^{\prime}$ to an $\mathrm{FH}_{5}$-coloring $c$ of $G$.

Let $c\left(v_{7} v\right)=F, c\left(w_{l} v_{l}\right)=c^{\prime}\left(w_{l} x\right)$ for $l \in\{i, j\}$, while $c\left(w_{l} v_{l}\right)=F$ and $c\left(v_{l} v\right)=H$ for $l \in\{1, \ldots, 6\}-\{i, j\}$. It remains to color $v_{i} v$ and $v_{j} v$. 
If at least one of $w_{i} v_{i}, w_{j} v_{j}$, say the first, is colored with $H$, then we let $c\left(v_{i} v\right)=F, c\left(v_{j} v\right)=H$. Suppose both $w_{i} v_{i}$ and $w_{j} v_{j}$ are colored with $F$. This means that $w_{i}$ and $w_{j}$ are not connected by an $F$-path in $G_{i, j}-x$, which implies that $v_{7}$ is not connected by an $F$-path in $G_{i, j}-x$ either with $w_{i}$ or with $w_{j}$. Suppose with the first; then we let $c\left(v_{i} v\right)=F$ and $c\left(v_{j} v\right)=H$.

It remains to assume that every pair of vertices in the set $\left\{w_{1}, \ldots, w_{6}\right\}$ has a common neighbor. Then we make use of the following claim.

Claim 9. Let $G^{\prime}$ be obtained from $G$ by deleting $v$ and all its neighbors of degree 2. If in $G^{\prime}$, every pair of vertices from the set $\left\{w_{1}, \ldots, w_{6}\right\}$ has a common neighbor, then there exists a vertex $x$ distinct from these six vertices such that $x$ is adjacent to all of them.

Proof. The proof is similar to that of Claim 8. In particular, Case 3 is identical and is omitted from the proof below. The only remark on Case 1 is that $w_{6} w_{3} \notin E(G)$, which implies that a strong face at $v$ is not adjacent to the triangle at $v$ and therefore saves $\frac{1}{2}$ for $v$. Case 2 is a little bit different and given in full detail below.

Case 2. There is no chord of the kind $w_{i} w_{i+3}$, but there is a chord $w_{i} w_{i+2}$. Since $w_{i+1}$ has a common neighbor with a $w_{i+4}$, we can assume by symmetry that $w_{i} w_{i+4} \in E(G)$. Note that $w_{i+3}$ can reach $w_{i+5}$ only through $w_{i+4}$ (which means that $w_{i+3} w_{i+4} \in E(G)$ ) and $w_{i+1}$ only through $w_{i+2}$ (which means that $w_{i+3} w_{i+2} \in E(G)$ ). This yields a 4 -cycle $w_{i} w_{i+2} w_{i+3} w_{i+4}$, a contradiction.

By the same reasoning as in the proof of Configuration 1, at least one of the edges $w_{2} w_{3}$ or $w_{3} w_{4}$, say the first, is not present in $G$. Then the face incident with $v, v_{2}$ and $v_{3}$ is strong. This face is not adjacent to the triangle at $v$ and has at least two vertices of degree 2, which implies that $v$ gives $\frac{1}{4}$ to each of them.

Configuration 3. Vertex $v$ is adjacent to five 2-vertices and incident with two triangles, where each triangle contains one of these five 2-vertices.

W.l.o.g., we can assume that $d\left(v_{1}\right)>2$ and there is a triangle $v v_{1} v_{2}$ at $v$. There are two cases to consider as shown in Figure 1.

(A) $d\left(v_{6}\right)>2$ and there is a triangle $v v_{5} v_{6}$, and

(B) $d\left(v_{7}\right)>2$ and there is a triangle $v v_{6} v_{7}$. 

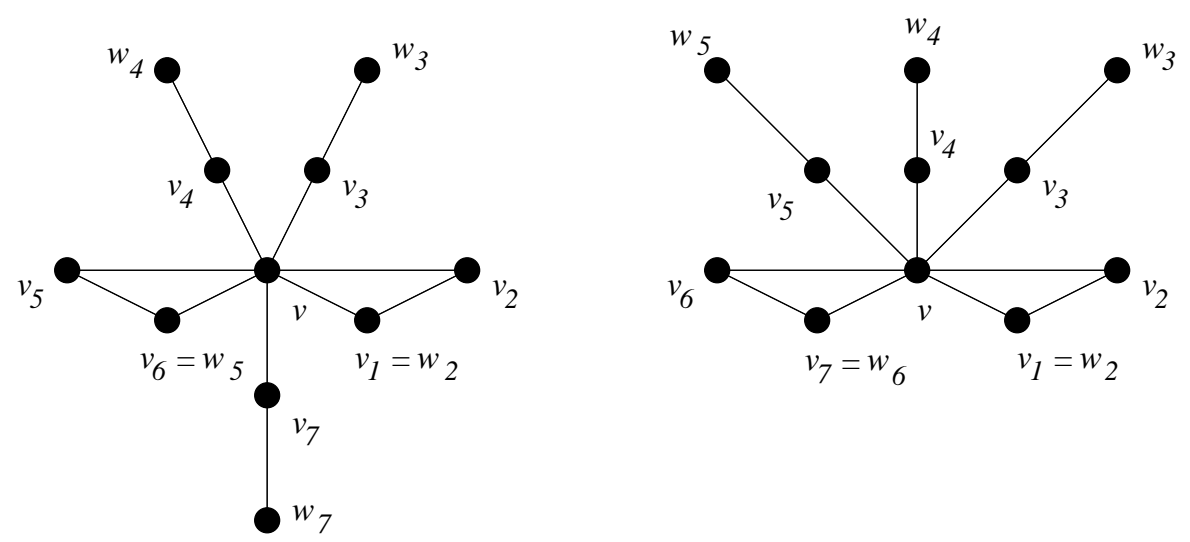

Figure 1. Two cases of Configuration 3.

Our argument below makes no distinction between (A) and (B), except in notation. For $i \in\{2, \ldots, 7\}$, if $d\left(v_{i}\right)=2$, then denote the vertex adjacent to $v_{i}$ other than $v$ by $w_{i}$. In particular, $w_{2}=v_{1}$ and either $w_{5}=v_{6}($ Case $(\mathrm{A}))$ or $w_{6}=v_{7}$ (Case (B)). We delete all 2-vertices adjacent to $v$ and add one of the following 2-paths to obtain a graph $G^{\prime}$ : either $v_{1} x_{1} w_{4}$ or $w_{3} x_{3} v_{6}$ in (A), and either $v_{1} x_{1} w_{4}$ or $w_{3} x_{3} v_{7}$ in (B). Note that vertex $x_{1}$ or $x_{3}$, as the case may be, is a newly-added vertex.

Note that $G^{\prime}$ has fewer branching vertices than $G$. First suppose $G^{\prime}$ is $C_{4}$-free; then let $c^{\prime}$ be an $F H_{5}$-coloring of $G^{\prime}$. We use $c^{\prime}$ to design an $F_{5^{-}}$ coloring $c$ of $G$. To fix notation, we give the argument for the case when $G^{\prime}$ contains 2-path $v_{1} x_{1} w_{4}$.

We define an intermediate coloring $c_{0}$ of $G$ and then adjust it to obtain a desired coloring $c$. Let $e^{*}$ be the edge other than $v v_{1}$ joining $v$ with a vertex of degree greater than 2, i.e., $e^{*}=v v_{6}$ in $(\mathrm{A})$ and $e^{*}=v v_{7}$ in (B). Let $c_{0}\left(v v_{1}\right)=c^{\prime}\left(v v_{1}\right)$ and $c_{0}\left(e^{*}\right)=c^{\prime}\left(e^{*}\right)$. Also for each $i$ such that $d\left(v_{i}\right)=2$, we let $c_{0}\left(v v_{i}\right)=H$ and $c_{0}\left(v_{i} w_{i}\right)=F$.

If $c_{0}\left(v v_{1}\right)=c_{0}\left(e^{*}\right)=F$ then $c_{0}$ is already the desired $F H_{5}$-coloring $c$ of $G$. We are also easily done if $c_{0}\left(v v_{1}\right)=c_{0}\left(e^{*}\right)=H$ by letting $c\left(v v_{1}\right)=$ $c\left(v v_{2}\right)=F$ and $c\left(v_{1} v_{2}\right)=H$.

Suppose $c_{0}\left(v v_{1}\right)=F$ and $c_{0}\left(e^{*}\right)=H$. At this point, $c_{0}$ is not an $F H_{5^{-}}$ coloring as there are six edges incident to $v$ that are colored $H$. We will recolor some edges to obtain $F H_{5}$-coloring $c$. In the case that $c^{\prime}\left(v_{1} x_{1}\right)=H, c$ is obtained by recoloring $v v_{2}$ with $F$ and $v_{1} v_{2}$ with $H$. Now if $c^{\prime}\left(x_{1} w_{4}\right)=H$, 
we let $c\left(v_{4} w_{4}\right)=H$ and $c\left(v v_{4}\right)=F$. So we can assume that $c^{\prime}\left(v_{1} x_{1}\right)=$ $c^{\prime}\left(x_{1} w_{4}\right)=F$; now it suffices to let $c\left(v v_{4}\right)=F$.

Finally, suppose that $c_{0}\left(v v_{1}\right)=H$ and $c_{0}\left(e^{*}\right)=F$. The case $c^{\prime}\left(x_{1} w_{4}\right)=$ $H$ is resolved as in the previous paragraph. If $c^{\prime}\left(v_{1} x_{1}\right)=H$, we can let $c\left(v_{1} v_{2}\right)=H$ and $c\left(v v_{2}\right)=F$. So we can assume that $c^{\prime}\left(v_{1} x_{1}\right)=c^{\prime}\left(x_{1} w_{4}\right)=$ $F$. This implies that there is no $F$-path from $v_{1}$ to $w_{4}$. Now the end of $e^{*}$ other than $v$ is not joined by an $F$-path either with $v_{1}$ or with $w_{4}$, and it suffices to let $c\left(v v_{1}\right)=F$ or $c\left(v v_{4}\right)=F$, respectively.

Thus, our initial supposition that $G^{\prime}$ is $C_{4}$-free has been ruled out. So we have that $G$ has a 2-path joining $v_{1}$ with $w_{4}$ (in order for there to be a $C_{4}$ in $G^{\prime}$ ). The same argument, with a slight difference in notation, shows that $G$ has a 2-path joining $w_{3}$ with $v_{6}$ if (A) happens and with $v_{7}$ otherwise. This means that $G$ has a vertex $w$ adjacent to $v_{1}, w_{3}, w_{4}$, and either $v_{6}$ (if (A) is the case) or $v_{7}$ (otherwise).

If the face $f_{3}=v_{3} v v_{4} \ldots$ is strong, then $v$ gives only $\frac{1}{4}$ to each of $v_{1}$ and $v_{2}$, and $\mu^{*}(v) \geq 7-4-5 \times \frac{1}{2}-2 \times \frac{1}{4}=0$. If, on the other hand, face $f_{3}$ is weak, then, since $d(w)>2$ and there is a path $w_{3} w w_{4}$, it follows that $f_{3}$ must be a 5 -face incident with edge $q w_{3} w_{4}$. In that case, the faces $f_{2}=v_{2} v v_{3} \ldots$ and $f_{4}=v_{4} v v_{5} \ldots$ are strong since edges $w_{2} w_{3}$ and $w_{4} w_{5}$ cannot exist, and again $\mu^{*}(v) \geq 0$.

Configuration 4. Vertex $v$ is adjacent to four 2-vertices and incident with three triangles, each containing one of these 2 -vertices.

Let $v$ be adjacent to 2-vertices $v_{2}, v_{4}, v_{6}$ and $v_{7}$, and the three triangles are $v v_{1} v_{2}, v v_{3} v_{4}$ and $v v_{5} v_{6}$. Furthermore, let $w_{7}$ be the neighbor of $v_{7}$ other than $v$.

Let $G^{\prime}$ be the graph obtained by removing $v$ and 2-vertices adjacent to $v$, adding vertices $z_{1}, z_{3}$, and $z_{5}$, and adding the edges of the 6-cycle $C^{+}=v_{1} z_{1} v_{3} z_{3} v_{5} z_{5}$. Note that $G^{\prime}$ is $C_{4}$-free since the common neighbor, $v$, of $v_{1}, v_{3}, v_{5}$ in $G$ has been removed.

Since $G^{\prime}$ has fewer branching vertices than $G$, it is $F H_{5}$-colorable. Let $c^{\prime}$ be an $F H_{5}$-coloring of $G^{\prime}$. We use $c^{\prime}$ to design an $F H_{5}$-coloring $c$ of $G$ as follows.

Again, we first define an intermediate coloring $c_{0}$ and then we adjust it to obtain a desired coloring $c$. Let $c_{0}\left(v v_{i+1}\right)=c_{0}\left(v v_{7}\right)=H$, and $c_{0}\left(v_{i} v_{i+1}\right)=$ $c_{0}\left(v_{7} w_{7}\right)=F$ whenever $i \in\{1,3,5\}$. Also for $i \in\{1,3,5\}$, we let $c_{0}\left(v v_{i}\right)=$ $H$ if $v_{i}$ is incident with at least one edge of $C^{+}$colored $H$ (in coloring $c^{\prime}$ ); otherwise, we let $c_{0}\left(v v_{i}\right)=F$. 
Note that at least one of $v v_{1}, v v_{3}$ and $v v_{5}$ is colored $H$ since $C^{+}$cannot be totally colored with $F$ in coloring $c^{\prime}$. Thus, we have three cases to consider.

Case 1. Exactly one of the edges $v v_{1}, v v_{3}$ and $v v_{5}$ is colored $H$. Without loss of generality, suppose $c_{0}\left(v v_{1}\right)=H, c_{0}\left(v v_{3}\right)=c_{0}\left(v v_{5}\right)=F$. Here, $c_{0}$ is already a desired $\mathrm{FH}_{5}$-coloring $c$ of $G$ since $G-v$ has no $F$-path from $v_{3}$ to $v_{5}$ due to the absence of $F$-cycles in $G^{\prime}$ going through $v_{3} z_{3}$ and $z_{3} v_{5}$.

Case 2. Exactly two of the edges $v v_{1}, v v_{3}$ and $v v_{5}$ are colored $H$. Thus, $c_{0}$ is not an $\mathrm{FH}_{5}$-coloring (as there are six edges incident to $v$ that are colored $H$ in $c_{0}$ ). We will recolor some edges to yield an $F H_{5}$-coloring $c$.

Without loss of generality, suppose $c_{0}\left(v v_{1}\right)=c_{0}\left(v v_{3}\right)=H, c_{0}\left(v v_{5}\right)=F$. By our construction of $c_{0}$ from $c^{\prime}$, this implies that $c^{\prime}\left(z_{3} v_{5}\right)=c^{\prime}\left(v_{5} z_{5}\right)=F$ in $G^{\prime}$, and at least one edge of $C^{+}$incident to $v_{1}$ is colored $H$ in $c^{\prime}$ and similarly at least one edge of $C^{+}$incident to $v_{3}$ is colored $H$ in $c^{\prime}$. This yields three subcases to consider.

Subcase 2.1. Both the edges of $C^{+}$incident to one of $v_{1}$ or $v_{3}$ are colored $H$ : say, $c^{\prime}\left(v_{1} z_{1}\right)=c^{\prime}\left(v_{1} z_{5}\right)=H$. To obtain $c$, swap the colors on the edges $v v_{2}$ and $v_{1} v_{2}$ (similarly, swap the colors on edges $v v_{4}$ and $v_{3} v_{4}$, if it had been $v_{3}$ whose both edges in $C^{+}$were colored $H$ ).

So, now we are left with two subcases where each of $v_{1}$ and $v_{3}$ has exactly one of their $C^{+}$edges colored $F$ and one colored $H$.

Subcase 2.2. One of the edges of $C^{+}$at $v_{1}$ or $v_{3}$ colored $F$ is incident with one of the two edges of $C^{+}$at $v_{5}$ (both of are which colored $F$ in the case under consideration): suppose, without loss of generality, that $c^{\prime}\left(v_{1} z_{5}\right)=F$. We recolor $v v_{2}$ with $F$ to obtain a desired $F H_{5}$-coloring $c$ of $G$ (since there is no $F$-cycle in $G^{\prime}$ going through $v_{1} z_{5}$ and $z_{5} v_{5}$, there is no $F$-cycle containing the $F$-path $v_{1} v_{2} v v_{5}$ in $G$ ).

Subcase 2.3. $c^{\prime}\left(v_{1} z_{5}\right)=c^{\prime}\left(v_{3} z_{3}\right)=H$, which means that $c^{\prime}\left(v_{1} z_{1}\right)=$ $c^{\prime}\left(v_{3} z_{1}\right)=F$. This implies the absence of an $F$-path from $v_{1}$ to $v_{3}$ in $c_{0}$. As a consequence, either there is no $F$-path from $v_{5}$ to $v_{1}$ or there is no $F$-path from $v_{3}$ to $v_{5}$. If the former is true, we recolor $v v_{2}$ with $F$ to obtain $c$; otherwise, we recolor $v v_{4}$ with $F$.

Case 3. All three of the edges $v v_{1}, v v_{3}$, and $v v_{5}$ are colored $H$ in $c_{0}$. In $c_{0}$, all the seven edges of $v$ are colored $H$. To obtain $c$, it suffices to recolor edges $v v_{1}$ and $v v_{2}$ with $F$, and recolor $v_{1} v_{2}$ with $H$. 
So, we have proved that the final charge of each 7 -vertex is non-negative. This completes the proof of Theorem 1.

\section{REMARKS}

The proof above shows that a planar $C_{4}$-free graph has one of the configurations that cannot exist in a minimal counterexample. Since these configurations can be identified in polynomial time (in terms of the number of vertices), our proof yields a polynomial time algorithm for decomposing a planar $C_{4}$-free graph into a forest and a graph of maximum degree at most 5 .

In the rest of this section, we construct a planar $C_{4}$-free graph that cannot be decomposed into a forest and a graph of maximum degree 3 . This leaves open the question whether every planar $C_{4}$-free graph can be decomposed into a forest and a graph of maximum degree at most 4 .

It is well-known that there are infinitely many cubic planar graphs with girth 5 , and the dodecahedron (having 20 vertices) is the smallest of them. Let $G$ be an $n$-vertex cubic planar graph with girth 5 , having $m$ edges and $f$ faces. We construct a planar graph $G^{\prime}$ from $G$ as follows:

(1) for every face $f$, add a vertex $v_{f}$ and connect $v_{f}$ with each vertex on the boundary of $f$ with an edge and then subdivide such edge with a new vertex;

(2) for every edge $e$ of the original graph $G$, add a vertex and join it by edges with both ends of $e$.

Denote the numbers of vertices, edges, faces, and vertices of degree 2 in $G^{\prime}$ by $n^{\prime}, m^{\prime}, f^{\prime}$ and $n_{2}^{\prime}$, respectively. By construction,

$$
\begin{gathered}
n^{\prime}=n+(f+2 m)+m=n+3 \times \frac{3}{2} n+\frac{1}{2} n+2=6 n+2, \\
m^{\prime}=3 m+4 m=7 m=\frac{21}{2} n, \quad \text { and } \quad n_{2}^{\prime}=m+2 m=\frac{9}{2} n .
\end{gathered}
$$

Suppose $E\left(G^{\prime}\right)$ has a partition into a spanning tree $F$ and a subgraph $H$ such that $\Delta(H) \leq 3$. Since $F$ is a spanning tree we have:

(a) for every 2-vertex $v$ in $G^{\prime}, \operatorname{deg}_{H}(v) \leq 1$;

(b) for each face $f$ of $G$, at least one 2-vertex in $G^{\prime}$ adjacent to $v_{f}$ has two neighbors in $F$ and hence is isolated in $H$. 
Therefore, $\sum_{v \in V\left(G^{\prime}\right)} \operatorname{deg}_{H}(v) \leq 3\left(n^{\prime}-n_{2}^{\prime}\right)+n_{2}^{\prime}-f=3(6 n+2)-2 \times \frac{9}{2} n-\frac{1}{2} n-2$ $=\frac{17}{2} n+4$.

Hence, $m^{\prime}=|E(F)|+|E(H)| \leq n^{\prime}-1+\frac{1}{2}\left(\frac{17}{2} n+4\right)=\frac{41}{4} n+3<\frac{21}{2} n=m^{\prime}$, a contradiction for all $n \geq 14$.

\section{REFERENCES}

[1] A. Bassa, J. Burns, J. Campbell, A. Deshpande, J. Farley, M. Halsey, S. Michalakis, P.-O. Persson, P. Pylyavskyy, L. Rademacher, A. Riehl, M. Rios, J. Samuel, B. Tenner, A. Vijayasaraty, L. Zhao and D. J. Kleitman, Partitioning a planar graph of girth ten into a forest and a matching, manuscript (2004).

[2] O.V. Borodin, Consistent colorings of graphs on the plane, Diskret. Analiz 45 (1987) 21-27 (in Russian).

[3] O. Borodin, A. Kostochka, N. Sheikh and G. Yu, Decomposing a planar graph with girth nine into a forest and a matching, European Journal of Combinatorics 29 (2008) 1235-1241.

[4] O. Borodin, A. Kostochka, N. Sheikh and G. Yu, M-degrees of quadrangle-free planar graphs, J. Graph Theory 60 (2009) 80-85.

[5] W. He, X. Hou, K.W. Lih, J. Shao, W. Wang and X. Zhu, Edge-partitions of planar graphs and their game coloring numbers, J. Graph Theory 41 (2002) 307-317.

[6] D.J. Kleitman, Partitioning the edges of a girth 6 planar graph into those of a forest and those of a set of disjoint paths and cycles, manuscript.

Received 27 November 2007

Revised 1 August 2008

Accepted 29 August 2008 